\title{
Atomic Pair-State Interferometer: Controlling and Measuring an Interaction-Induced Phase Shift in Rydberg-Atom Pairs
}

\author{
J. Nipper, J. B. Balewski, A. T. Krupp, S. Hofferberth, R. Löw, and T. Pfau \\ 5. Physikalisches Institut, Universität Stuttgart, Pfaffenwaldring 57, 70569 Stuttgart, Germany
}

(Received 20 February 2012; published 15 August 2012)

\begin{abstract}
We present experiments measuring an interaction-induced phase shift of Rydberg atoms at Stark-tuned Förster resonances. The phase shift features a dispersive shape around the resonance, showing that the interaction strength and sign can be tuned coherently. We use a pair-state interferometer to measure the phase shift. Although the coupling between pair states is coherent on the time scale of the experiment, a loss of visibility occurs as a pair-state interferometer involves three simultaneously interfering paths and only one of them is phase shifted by the mutual interaction. Despite additional dephasing mechanisms, a pulsed Förster coupling sequence allows for observation of coherent dynamics around the Förster resonance.
\end{abstract}

DOI: 10.1103/PhysRevX.2.031011

Subject Areas: Atomic and Molecular Physics, Biological Physics, Quantum Physics

Coherent control of strongly interacting gases is of great interest, as they can serve as model systems for correlated quantum many-body physics. Rydberg atoms are of particular interest in ultracold atomic physics because they offer strong interactions that are tunable both in strength and character. Noteworthy applications are, for example, quantum computing [1] or quantum simulation [2], as well as ultracold chemistry [3]. Coherent Rydberg interactions are expected not only to create exotic ground states and quantum phase transitions [4] but also to realize quantum dynamics, for example, in coherent energy transport in strongly correlated many-body systems [5,6]. In biophysics these nonradiative exciton dynamics are relevant for photosynthesis in certain biological systems [7] and might be modeled using strongly interacting ultracold atoms [8].

A promising tool for creation and control of strong interactions are based on Stark-tuned Förster resonances, where the strength and character of the atom-atom interactions, from resonant dipole-dipole interaction to van der Waals interaction, can be tuned by small electric fields [5,9-14]. Here, we present two kinds of pulsed experiments, following the idea of Ramsey experiments, that allow phase-sensitive detection and studies of coherent two-body dynamics at a Förster resonance by measuring the total number of Rydberg atoms only. In the first experiment, we observe oscillations in the visibility of the Ramsey fringes of a double-Ramsey-type sequence for Rydberg pair states, consisting of two laser excitation pulses and two electric-field pulses. This experiment shows that Rydberg-atom pairs close to a Förster resonance evolve coherently, a prerequisite for an interaction-induced phase shift used in proposals for Rydberg quantum gates

Published by the American Physical Society under the terms of the Creative Commons Attribution 3.0 License. Further distribution of this work must maintain attribution to the author(s) and the published article's title, journal citation, and DOI.
$[1,15]$. In the second experiment, we measure this tunable phase shift using a pair-state interferometer.

Previously, coherence at Förster resonances in Rydberg systems has been studied by means of Ramsey techniques in the coupling between pair states $[16,17]$ and by direct observation of Rabi oscillations between the ground and Rydberg states [18]. A decreased coherence time at resonance was measured and quantified based on two-level optical Bloch equations in [17].

To describe the coherent dynamics we observe in both measurements, we introduce in this paper the concept of a pair-state Ramsey interferometer, extending the usual twolevel atom interferometer to two interacting many-level atoms coupled to the optical excitation field. Besides the ground state $|g g\rangle$ and the doubly excited state $|r r\rangle$, there are two singly excited states $|g r\rangle$ and $|r g\rangle$. Only the symmetric combination of the two couples to the Ramsey field. Therefore the pair-state version of a Ramsey interferometer consists of three simultaneously interfering paths. Only one of them is affected by the possibly coherent Rydberg-Rydberg interaction $U$ and experiences a phase shift $\varphi(U)$. The Hamiltonian describing this pairstate interferometer can be found in the Appendix.

Figure 1(a) shows a schematic of the relevant pair states for a Ramsey interferometer in an ensemble of atoms with switchable interaction. The interferometer starts with both atoms in the ground state $|g g\rangle$. Optical light pulses excite atoms to the Rydberg state and induce a coupling between $|g g\rangle,(|g r\rangle+|r g\rangle) / \sqrt{2}$, and $|r r\rangle$. A switchable interaction can be induced by coupling $|r r\rangle$ to another, previously unpopulated Rydberg-atom pair state $\left|r^{\prime} r^{\prime \prime}\right\rangle$. Here, a Stark-tuned Förster resonance is used, but the concept of the pair-state interferometer is valid for any tunable interaction. In the case of weak interactions (adiabatic regime), a phase shift of $|r r\rangle$ occurs. Strong interactions (diabatic regime) induce a nonadiabatic mixing of the pair states and lead to a population of $\left|r^{\prime} r^{\prime \prime}\right\rangle$. The total number of Rydberg atoms, depending on the populations both in the doubly 


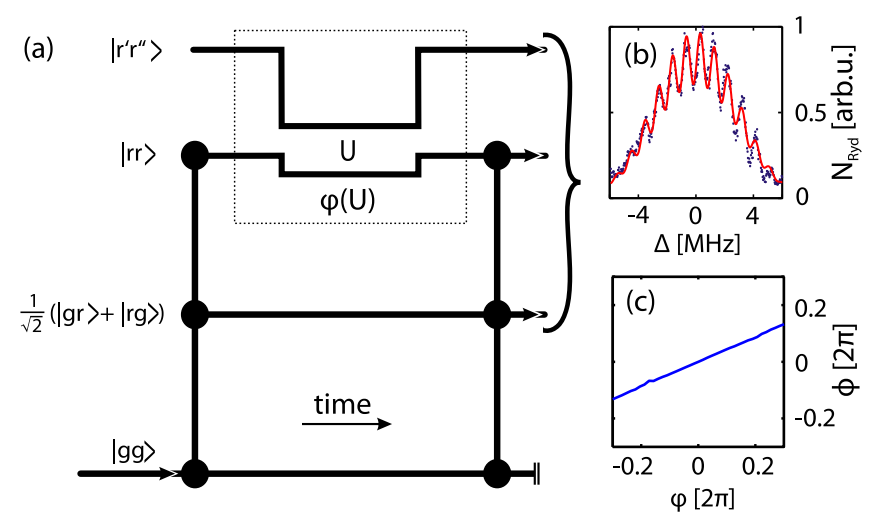

FIG. 1. (a) Schematic of the pair-state interferometer. Interactions between Rydberg atoms change the phase $\varphi(U)$ of the $|r r\rangle$ state relative to the other states and create population in $\left|r^{\prime} r^{\prime \prime}\right\rangle$. A pulsed Ramsey field couples three states simultaneously. A Rydberg detector detects the number of Rydberg atoms $N_{\text {Ryd }}$, resulting in (b) a Ramsey spectrum (blue data points) depending on the combined detuning $\Delta$ of the twophoton excitation. The red line is a fit to the data. (c) The transfer function for $0.6 \pi$-Ramsey pulses describing the dependence of the fitted phase of the Ramsey fringes $\phi$ on the phase shift $\varphi$ in the $|r r\rangle$ path for small angles.

excited pair states $|r r\rangle$ and $\left|r^{\prime} r^{\prime \prime}\right\rangle$ and in the singly excited pair state $(|g r\rangle+|r g\rangle) / \sqrt{2}$, is detected. From a fit to the Ramsey spectrum in frequency space [Fig. 1(b)], the visibility and the phase $\phi$ of the Ramsey fringes can be obtained (see the Appendix).

This four-path interferometer behaves considerably differently than a two-path interferometer. For example, even in the case of adiabatically switched interactions and an individual pair of atoms, a coherent phase shift $\varphi(U)$ in the $|r r\rangle$ path leads to a loss of visibility that cannot be avoided. This is one source of the reduced coherence times observed in Ramsey experiments [17]. Similar effects have also been observed in atom interferometry [19]. Additionally, the phase shift $\varphi(U)$ translates to a phase shift $\phi$ of the Ramsey fringes. The transfer function from $\varphi(U)$ to $\phi$ is a nontrivial function, depending on the populations of the different paths of the interferometer. Figure 1(c) shows such a simulated transfer function for $0.6 \pi$-Ramsey pulses. For small angles it is a monotonic function in $\varphi(U)$. Close to $\varphi(U)=\pi$, the fringe pattern is strongly disturbed and the phase $\phi$ is not well defined. Under any circumstances the effective phase shift of the Ramsey fringes $\phi$ is smaller than the phase shift of the doubly excited state $\varphi$.

In an ensemble with strong binary interactions the ensemble average over different interaction strengths due to the distance and angular dependence of the interaction even at constant density results in an additional dephasing. Moreover, an inhomogeneous density distribution will lead to yet another source of dephasing. Furthermore, in the experiment decoherence due to a finite excitation linewidth

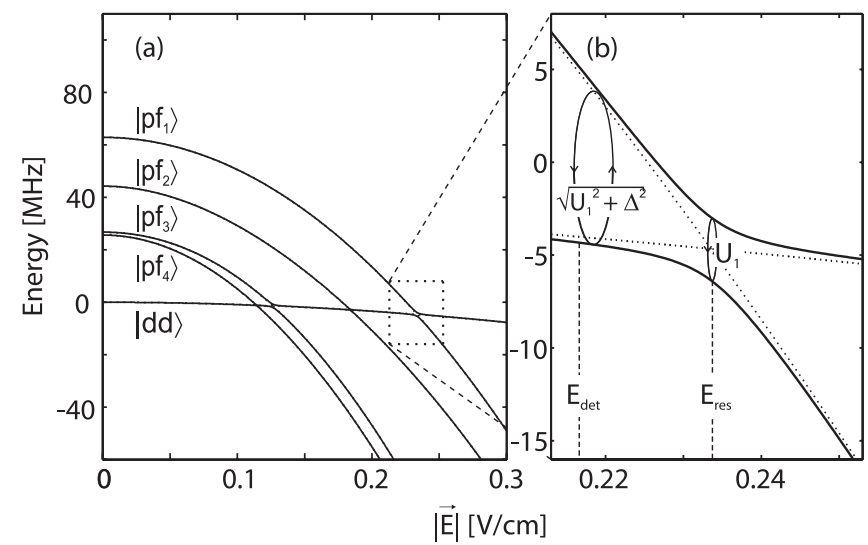

FIG. 2. (a) Stark map of the relevant pair states (see text). (b) A magnification of the Stark map at the electric fields where the pair states are tuned into resonance. The dotted lines denote the pair-state energies without coupling; the solid lines include the dipole-dipole coupling for an interatomic distance of $9 \mu \mathrm{m}$. The energy differences at the resonant electric field $E_{\text {res }}$ and at a detuned electric field $E_{\text {det }}$ are indicated.

is present. Previous measurements so far cannot separate these different sources. However, echo or Ramsey-type sequences can refocus some of the inhomogeneous dephasing and allow for the observation of coherent evolution of the ensemble in our first experiment.

The Stark-tuned Förster resonances appear if two dipole-dipole-coupled pair states are shifted into resonance by a small applied electric field. Here we employ Förster resonances in ${ }^{87} \mathrm{Rb}$ between the pair states $2 \cdot 44 d_{5 / 2}$ and $46 p_{3 / 2}+42 f_{7 / 2}$, denoted by $|d d\rangle$ and $\left|p f_{i}\right\rangle$, respectively. In the pair-state interferometer these states correspond to the pair states $|r r\rangle$ and $\left|r^{\prime} r^{\prime \prime}\right\rangle$. Different magnetic substates of the $42 f$ state lead to several resonances at slightly different electric fields, indicated by the subscript $i$. The pair state $\left|p f_{1}\right\rangle$ is the stretched configuration including the magnetic substate $42 f_{7 / 2}, m_{j}=7 / 2$. The other magnetic substates are not eigenstates of $\hat{J}_{z}$ in the combined electric and magnetic field. Figure 2 shows the Stark shift of the pair states in a 13.55-G magnetic offset field, parallel to the electric field. The Stark shifts were calculated by diagonalizing the single-atom Hamiltonian taking the magnetic and electric fields into account [17]. In a zero electric field, a finite Förster defect $\Delta=$ $E_{\left|p f_{i}\right\rangle}-E_{|d d\rangle}$, the energy difference between the coupled pair states, is present. With an increasing electric field the pair states experience different Stark shifts, and the Förster defect can be tuned [Fig. 2(a)]. A high magnetic field, as shown here, is advantageous as it increases the splitting between the pair states and simplifies the task of addressing a single Förster resonance.

At a sufficiently large $\Delta$ the interaction between the atoms can be calculated from second-order perturbation theory, and one obtains a van der Waals interaction energy of 


$$
\Delta E_{\mathrm{vdW}} \approx-\frac{\left|U_{i}\right|^{2}}{\Delta}
$$

for every dipole-coupled pair state $i . U_{i}$ is the strength of the dipole-dipole coupling, given by

$$
U_{i}(r, \Theta)=\sqrt{2} \cdot\left\langle p f_{i}\left|V_{d d}(r, \Theta)\right| d d\right\rangle,
$$

where $V_{d d}=\frac{\vec{p}_{1} \cdot \vec{p}_{2}-3\left(\vec{n} \cdot \vec{p}_{1}\right)\left(\vec{n} \cdot \vec{p}_{2}\right)}{r^{3}}$ is the dipole-dipole operator, $r$ is the interatomic distance, and $\vec{p}_{1,2}$ are the electric-dipole moments of the atoms. The $\sqrt{2}$ factor stems from the degeneracy of $\left|p f_{i}\right\rangle$ and $\left|f_{i} p\right\rangle$. This interaction increases with decreasing Förster defect, and the interaction strength can be tuned by the electric field. For $\Delta=0$, resonant dipoledipole interaction occurs, resulting in the Förster resonance, and the two pair states form an avoided crossing, as shown in Fig. 2(b). For small Förster defects $\Delta \approx U_{i}$, the eigenstates have to be obtained by diagonalization.

The strength of the dipole-dipole coupling $U_{i}(r, \Theta)$ is calculated as in $[9,17]$. The experiments are performed in an extended sample; thus the angular dependency is averaged out. For this paper, interactions at finite Förster defect $\Delta$ are most relevant where the interaction is dominated by the van der Waals interaction. Therefore the quadratic mean of the angle-dependent interaction is calculated. This gives values of $U_{1}(r)=719 \mathrm{MHz} \cdot \mu \mathrm{m}^{3} / r^{3}, U_{2}(r)=$ $200 \mathrm{MHz} \cdot \mu \mathrm{m}^{3} / r^{3}, \quad U_{3}(r)=654 \mathrm{MHz} \cdot \mu \mathrm{m}^{3} / r^{3}, \quad$ and $U_{4}(r)=167 \mathrm{MHz} \cdot \mu \mathrm{m}^{3} / r^{3}$ for the resonances at $0.23 \mathrm{~V} / \mathrm{cm}, 0.18 \mathrm{~V} / \mathrm{cm}, 0.13 \mathrm{~V} / \mathrm{cm}$, and $0.12 \mathrm{~V} / \mathrm{cm}$, respectively.

The experiments are performed in a magnetically trapped and evaporatively cooled cloud in the $f=2, m_{f}=2$ state. After cooling, the magnetic offset field is ramped to the highest experimentally realizable strength of $13.55 \mathrm{G}$. This results in an atomic cloud of about $700 \mathrm{nK}$ temperature at a peak density of about $2 \cdot 10^{12} / \mathrm{cm}^{3}$. The size of the atomic cloud in this high offset field reaches $1 / e$ radii of a Gaussian density distribution of $114 \mu \mathrm{m}$ longitudinally and $22 \mu \mathrm{m}$ radially. Further information about the experimental setup can be found in [20].

Rydberg atoms are excited by two-photon excitation via the $5 p_{3 / 2}$ state, blue detuned by $400 \mathrm{MHz}$ from the intermediate state to preserve the coherence in the excitation process. The total laser linewidth of the two-photon transition is below $2 \pi \times 100 \mathrm{kHz}$, and the single-atom Rabi frequency is about $\Omega_{0}=2 \pi \times 25 \mathrm{kHz}$. Optical Ramsey spectroscopy is realized throughout this paper by two short laser pulses of $\tau_{p}=150 \mathrm{~ns}$ duration, separated by a delay time of $\tau_{\text {del }}=800 \mathrm{~ns}$, as shown in Fig. 3(b). The pulse area is small enough that the system is not driven into saturation, but a collective enhancement of the excitation occurs [21]. After this pulse sequence the Rydberg atoms are field ionized and detected in an ion detector. As the field ionization is not state selective, the total Rydberg-atom number $N_{\text {Ryd }}$ is measured, independent of the Rydberg states that the atoms populate. Including a delay time to allow the

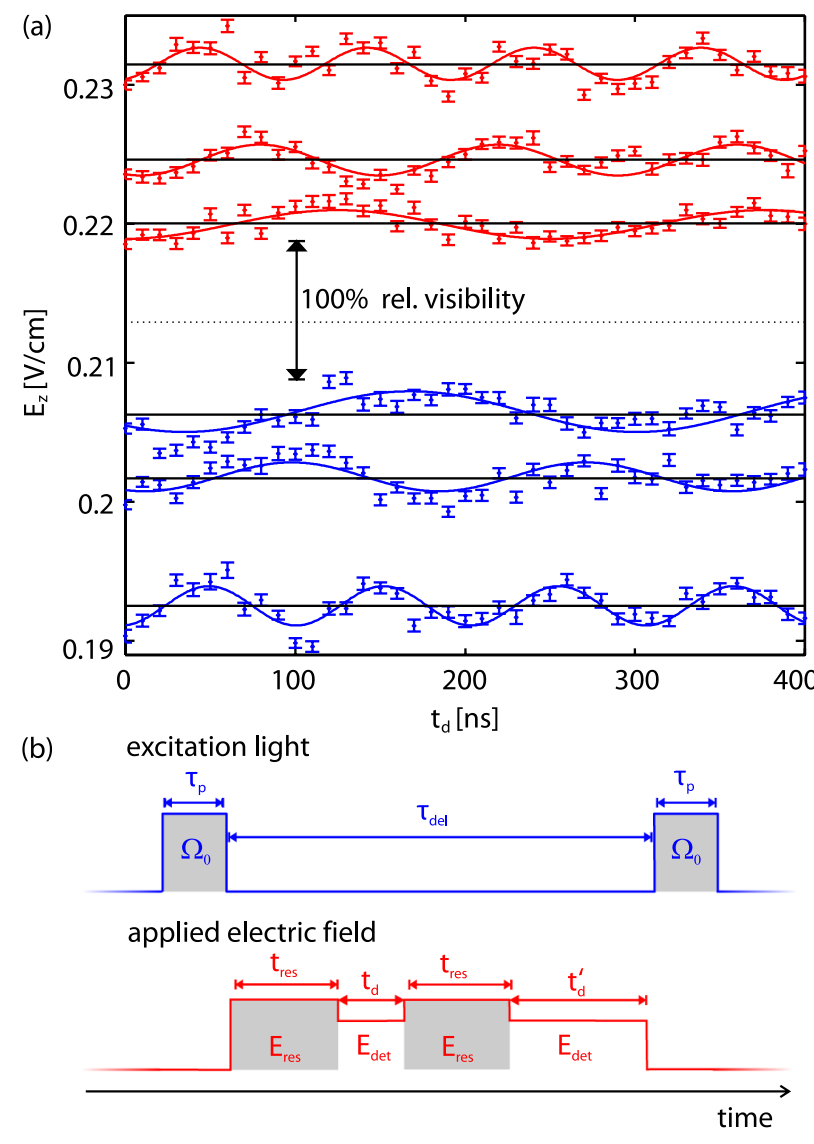

FIG. 3. (a) Oscillations in the visibility are measured for (b) the pulse sequence of the double-Ramsey experiment. The electric field during the delay time $E_{\text {det }}$ is indicated by the solid horizontal black lines in (a). The oscillations in the visibility (data points with error bars resulting from the standard deviation of the fit to the Ramsey spectrum) are centered around the applied electric field. The solid curves are sinusoidal fits to the data. Blue data (lower three measurements) and red data (upper three measurements) indicate positive and negative Förster defects, respectively. The dotted line indicates the position of the Förster resonance.

strong electric fields for the field ionization to decay one sequence of excitation and detection takes $8 \mathrm{~ms}$ and is repeated in one atomic sample 401 times. Thereby a whole spectrum ranging from -6 to $+6 \mathrm{MHz}$ around the atomic resonance is measured in one atomic sample without the need of averaging over different samples. As in Fig. 1(b), these Ramsey spectra show typical Ramsey fringes, which can be fitted to obtain the visibility $V$ and the phase $\phi$. The visibility in a pair-state interferometer is affected by a pair-interaction phase shift $\varphi(U)$, by population transfer between the Rydberg pair states, and by dephasing and decoherence processes. The phase of the Ramsey fringes $\phi$ provides information about $\varphi(U)$ according to the transfer function.

Between the optical Ramsey pulses an electric field can be tuned, enhancing the interaction close to a Förster resonance during the delay time only. Thereby the interaction 
strength during the delay time can exceed the maximally allowed interaction strength due to Rydberg blockade during the excitation.

In an experiment close to the Förster resonance the $|p f\rangle$ state couples only to $|d d\rangle$ via electric-field-dependent dipole-dipole coupling, but it does not couple to the light field, realizing an interferometer as depicted in Fig. 1. This offers the possibility to study the coherent evolution of the subsystem $|d d\rangle,|p f\rangle$ (dashed-line box in Fig. 1) separately by applying a Ramsey-like electric-field sequence, similar to [16]. The pulse sequence for this double-Ramsey experiment can be found in Fig. 3(b) and is comparable to Ramsey experiments on Feshbach resonances [22], where a similar magnetic field sequence was used. Here, the electric field is first pulsed for $t_{\text {res }}=200 \mathrm{~ns}$ to the electric field $E_{\text {res }}$ to tune the pair states $\left|p f_{1}\right\rangle$ and $|d d\rangle$ into resonance. The experimentally obtained value of this field is $E_{z}=0.213 \mathrm{~V} / \mathrm{cm} \mathrm{[17].} \mathrm{The} \mathrm{slight} \mathrm{mismatch} \mathrm{to} \mathrm{the}$ theory presented in Figs. 2(a) and 2(b) results from an unknown radial electric offset field $E_{\mathrm{r}}$ on the order of $0.05 \mathrm{~V} / \mathrm{cm}$ and a slight misalignment between the magnetic field, defining the quantization axis, and the applied electric field. Only the electric field $E_{z}$ in direction of the magnetic field is calibrated in this experiment. The rise time of the electric field is about $20 \mathrm{~ns}$. After a variable delay time $t_{\mathrm{d}}$ between $0 \mathrm{~ns}$ and $400 \mathrm{~ns}$, a second $200 \mathrm{~ns}$ pulse at $E_{\text {res }}$ is applied. Between and after these two Ramsey-like pulses $\left(t_{\mathrm{d}}\right.$ and $t_{\mathrm{d}}^{\prime}$ ), the electric field is detuned from exact resonance to a value between $E_{\mathrm{det}}=0.181 \mathrm{~V} / \mathrm{cm}$ and $E_{\mathrm{det}}=0.241 \mathrm{~V} / \mathrm{cm}$.

This experiment can be regarded as a Ramsey-like experiment between the $|d d\rangle$ and $\left|p f_{1}\right\rangle$ states only. The first resonant electric-field pulse couples the $|d d\rangle$ state to the $\left|p f_{1}\right\rangle$ state, generating a coherence between these states. During the delay time $t_{\mathrm{d}}$, the electric field is detuned from exact resonance [Fig. 2(b)]. The atom pairs will oscillate between $|d d\rangle$ and $\left|p f_{1}\right\rangle$ with the oscillation frequency $\sqrt{U^{2}+\Delta^{2}}$. The second resonant electric-field pulse interferes with both pair states again. The maximum visibility in the Ramsey spectrum occurs at an electric field $E_{\text {det }}$, where after the electric-field pulse sequence the atom pairs are refocused into the $|d d\rangle$ state. The oscillations between the pair states are now visible as oscillations in the visibility of the Ramsey fringes when the delay time $t_{\mathrm{d}}$ is varied.

This particular sequence offers the advantage that the total times at the resonant electric field $\left(2 \times t_{\text {res }}\right)$ as well as at the detuned field $\left(t_{\mathrm{d}}+t_{\mathrm{d}}^{\prime}\right)$ are constant. Thereby additional losses of coherence, e.g., due to inhomogeneous atom distributions, reduce the visibility to a constant value throughout this experiment. Oscillations in the visibility when the delay time between the resonant pulses is varied can clearly be separated. Figure 3(a) shows a selection of such double-Ramsey experiments for different values of $E_{\text {det }}$, indicated by the solid black lines. Oscillations in the visibility are clearly visible and no damping can be observed, indicating that the two-body coupling between the pair states is coherent at least on the time scale of the experiments. The oscillation frequency is obtained from sinusoidal fits to the data. It shows a minimum at the position of the Förster resonance at $E_{z}=0.213 \mathrm{~V} / \mathrm{cm}$, as expected. For the resonance here, $E_{z} \gg E_{\mathrm{r}}$ is valid; therefore we can approximately compensate the radial field in the calculations by a constant offset of $0.018 \mathrm{~V} / \mathrm{cm}$ in the electric field. With this offset taken into account, the Förster defect can be calculated, given the calculated Stark shifts in Fig. 2. In Fig. 4, the measured oscillation frequency versus the calculated Förster defect is plotted. The oscillation frequency follows the Förster defect $\Delta$, as expected for $\Delta \gg U$. Close to the Förster resonance a deviation from the linear behavior is expected. However, there the amplitude of the oscillations is strongly reduced, and no signal could be obtained for Förster defects of $|\Delta| \lesssim 2 \mathrm{MHz}\left(E_{z} \approx 0.209 \ldots 0.217 \mathrm{~V} / \mathrm{cm}\right)$.

This is also true for a slightly different pulse sequence where the electric field is tuned to zero after the second Ramsey-like electric-field pulse. This sequence converges to a single-pulse experiment for $E_{\mathrm{det}} \rightarrow E_{\mathrm{res}}$, where only the total pulse length is varied. Instead of direct Rabi oscillations only an exponential loss of visibility could be observed, as predicted in [23].

Interaction strengths in the range of $2 \mathrm{MHz}$ are expected at interatomic distances of $7 \mu \mathrm{m}$, giving a lower limit on the average Rydberg-atom distance. This is a reasonable value as the Rydberg signal is obtained from the whole cloud, including the wings, averaging the interatomic distance to rather large values. We interpret this experiment as follows: Strong dephasing occurs when the evolution of the system is dominated by the interaction between the pair states, as this interaction energy forms a broad band due to the dependence on the interatomic distance [24]. However, the coupling between single pair states is coherent on the time scale of the experiment. This coherence leads to the observed oscillations in the interferometer if the time evolution is dominated by the spatially constant Förster defect.

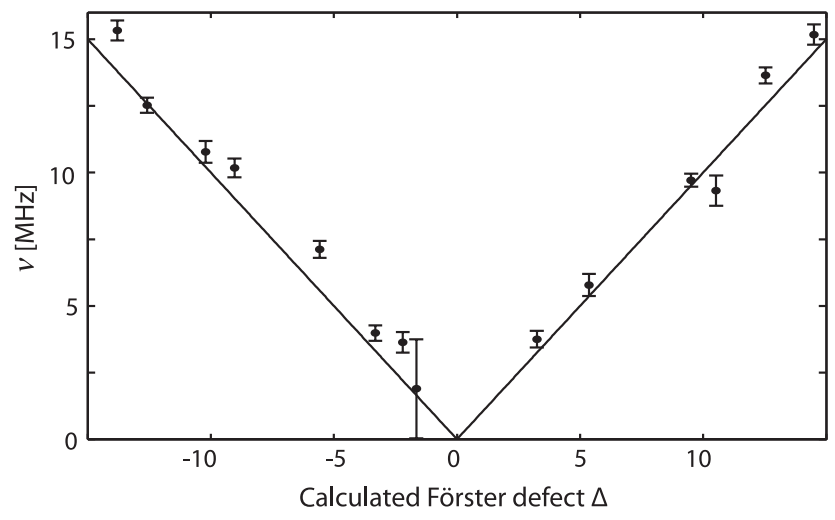

FIG. 4. Measured frequency $\nu$ of the oscillation in the visibility (data points) versus the calculated Förster defect. The error bars are the standard deviation of the sinusoidal fits. The solid line shows $\nu=|\Delta|$. 

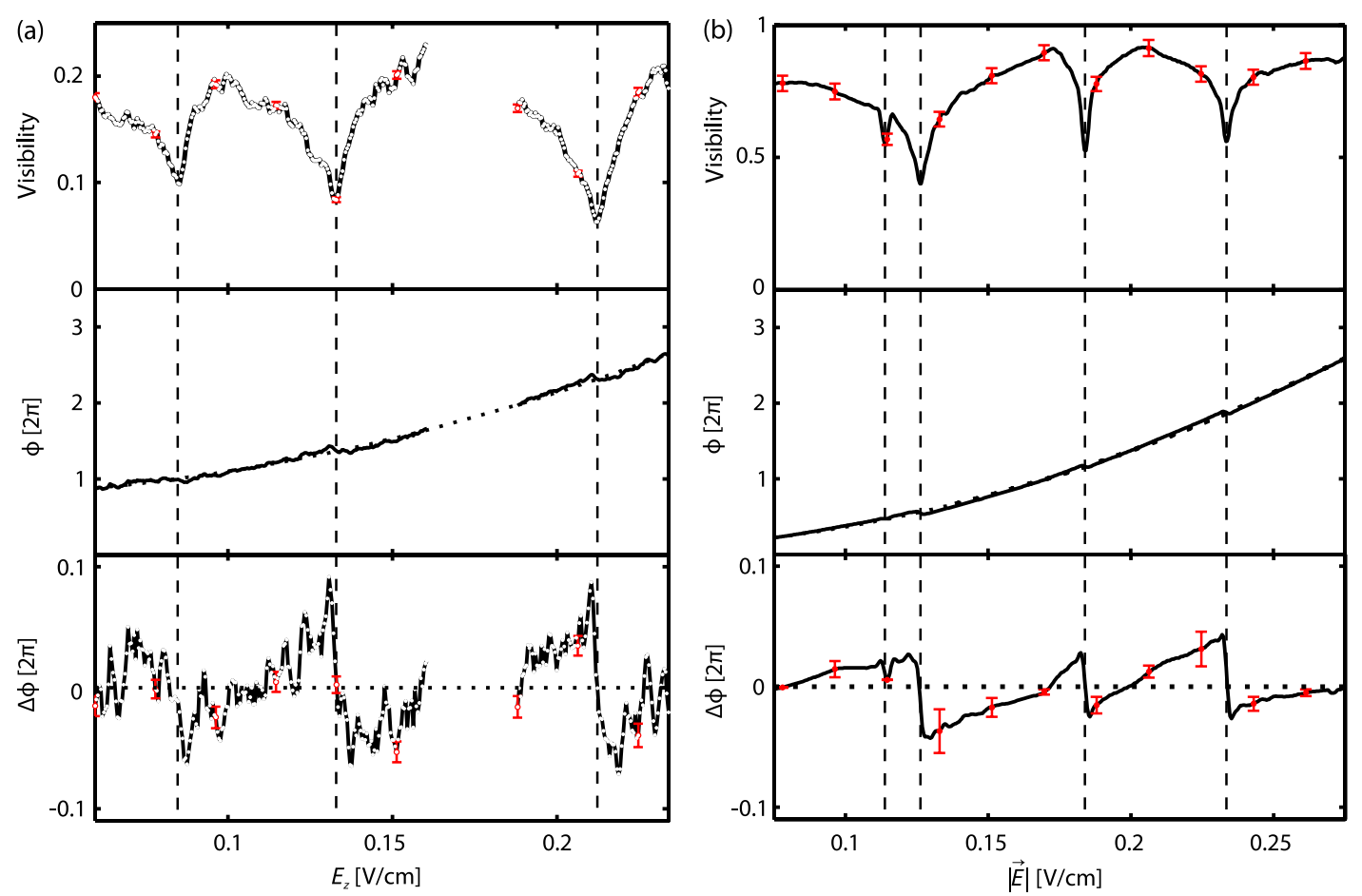

FIG. 5. Visibility and phase obtained from fits to (a) the measured and (b) the simulated Ramsey spectra versus the electric field. Note that the experimental spectra are plotted versus the calibrated component of the electric field $E_{z}$ and the simulated data versus the total electric field $|\vec{E}|$. The uppermost panels show the visibility, the middle panel shows the phase of the Ramsey fringes (solid line) and a quadratic fit to the data (dotted line), and the lower panels show the difference of the measured phase from the pure quadratic behavior. Some example error bars are plotted, denoting the standard deviation of the fit parameter.

Despite the fact that this coupling is coherent, it leads to a loss of visibility in the three-path pair-state interferometer. However, the phase shift $\varphi$ translates also to a measurable but small phase shift $\phi$ in the Ramsey fringe pattern. To study this, a second experiment is performed where we use a single electric-field pulse during the whole length of the delay time between the optical Ramsey pulses. The visibility and the phase of the measured Ramsey spectrum are obtained for varying strength $E_{z}$ of the pulsed electric field. Figure 5(a) shows the results of a fit to the measured Ramsey spectra. At the positions of the Förster resonances the visibility is reduced because of a population in $|p f\rangle$ and a phase shift $\varphi$ of $|d d\rangle$. Three distinct dips can be seen due to a substructure of the Förster resonance [17]. The phase shows a quadratic dependence as it is shifted by the quadratic Stark effect of the Rydberg atoms. If this pure quadratic effect is subtracted a deviation $\Delta \phi$ is visible that shows a clear dispersive behavior centered around the positions of the Förster resonances.

This interaction-induced phase shift can best be understood in the interferometer picture of Fig. 1. Here, the three states coupled by the light field are $|g g\rangle, \frac{1}{\sqrt{2}}(|g d\rangle+|d g\rangle)$, and $|d d\rangle$. A coupling between $|d d\rangle$ and $|p f\rangle$ leads to an interaction-induced phase shift of $|d d\rangle$ during the delay time, when the system is tuned close to the Förster resonance. For a first estimate, taking an interaction strength of $1 \mathrm{MHz}$ for two Rydberg atoms at Förster resonance and an interaction time of $0.8 \mu \mathrm{s}$, one would expect a phase shift of the pair state of $\varphi=0.8 \pi$. The transfer function of the pair-state interferometer (see Fig. 1) lowers the observed shift $\phi$. As the switching of the electric fields in the experiment is not adiabatic, a further reduction of $\phi$ is expected. The sign of the phase shift is determined by the direction of the interactioninduced energy shift of $|d d\rangle$, which changes sign at the resonance position, and a dispersive phase effect occurs.

This phase effect not only directly verifies the coherence of the interaction but also shows that the strength and the sign of the interaction can be tuned by the electric field. The interaction switches from attractive at electric fields smaller than the resonant field $E_{\text {res }}$ to repulsive above the resonance. Exactly on resonance two equally spaced states in the avoided crossing (Fig. 2) appear above and below the unperturbed states. Under these conditions the system is diabatically switched from the unperturbed $|d d\rangle$ state at $E=0 \mathrm{~V} / \mathrm{cm}$ to the perturbed states at $E=E_{\text {res }}$, generating a superposition state that does not experience interactions, and no phase shift occurs.

To model this experiment the Schrödinger equation is solved numerically for the experimental sequence, as described in the Appendix. Best results were obtained for an average distance of $9 \mu \mathrm{m}$, in good agreement with the estimated lower limit of $7 \mu \mathrm{m}$ from the double-Ramsey experiment, and for a Rabi frequency of $\Omega=2 \pi \times 2 \mathrm{MHz}$. 
This frequency is enhanced relative to the single-atom Rabi frequency by the collective excitation process. It is on the order of what is expected from simple estimates of the number of atoms per blockade sphere for a $44 d \mathrm{C}_{6}$ coefficient of $27 \mathrm{GHz} \cdot \mu \mathrm{m}^{6}$ at $|\vec{E}|=0 \mathrm{~V} / \mathrm{cm}$.

This model simplifies the actual system in many ways. It neglects the angular dependence of the interaction; only binary, next-neighbor interactions are calculated; and the many-body nature of the experiment is considered solely by a collective enhancement of the Rabi frequency. Nevertheless the model effectively allows us to understand the fundamental aspects of the experimental findings.

Figure 5(b) shows the calculated visibility and phase versus the total electric field $|\vec{E}|$. The absolute positions of the measured resonances are shifted relative to the calculations by uncontrolled radial electric fields. The visibility in the experiment is roughly a factor of 4 smaller than in the calculations. This mismatch might be explained by the finite laser linewidth. The minimal linewidth of the transition to the $44 d$ state at $700 \mathrm{nK}$ temperature was measured to be $300 \mathrm{kHz}$, broadened by the magnetic field gradient in the trap and possibly by electric-field inhomogeneities. This considerably reduces the visibility on the $1-\mu \mathrm{s}$ time scale of the experiment and is not included in the calculations. Furthermore, the measured dips are broader, possibly because of additional decoherence processes beyond the pair-state interferometer, e.g., resonant energy transfer in the many-body system [16]. However, qualitatively, the observed visibility at the Förster resonances can be reproduced with the two-body calculation as described above.

The phase, on the other hand, is not expected to be substantially disturbed by an additional loss of coherence and agrees remarkably well with the calculation. The dispersive shape of the signal and the amplitude are reproduced. Nevertheless, the phase of the Ramsey fringes is a nontrivial function of the populations of the pair states and the interaction strength. Thereby it strongly depends on the Rabi frequency and on the Rydberg-atom distribution. In a saturated ensemble, blockade effects clearly affect the nearestneighbor distribution, and many-body effects [16,25,26] occur beyond the two-body calculation. The inhomogeneous density in a trapped cloud will alter the distribution as well. This might account for the slight mismatch in Fig. 5.

In conclusion, we demonstrated the coherent coupling between pair states at a Förster resonance for Rydberg atoms, and we observed an interaction-induced phase shift on the atoms. The dispersive shape of the phase shift shows the tunability of the strength and the sign of the interaction. Fully coherent simulations of the introduced pair-state interferometer reproduce the observed phase shift and the loss in visibility.

In single-atom experiments [27], individual, single-siteaddressing $\pi$-excitation pulses can be used, and the system can be reduced to a two-path interferometer. There phase shifts on the order of $\pi$, as necessary for applications like phase gates, are realistic. Therefore we see these results as a step toward controlled phase gates [28] and quantum simulation, e.g., of energy transport processes [8] in quantum networks.

Because of the strong distance dependence of the interaction, Förster resonances can be used as a spectroscopic ruler [29]. This might offer a tool to gain more insight into the Rydberg-atom correlation function, which under certain conditions is expected to show a crystalline order [4].

We thank I. I. Ryabtsev for valuable discussions. This work is funded by the Deutsche Forschungsgemeinschaft (DFG) within the SFB/TRR21 and project PF 381/4-2. We also acknowledge support by the ERC under Contract No. 267100.

\section{APPENDIX: METHODS}

The pair-state interferometer in the case of one single Stark-tuned Förster resonance can be described in the basis $\left[|g g\rangle, \frac{1}{\sqrt{2}}(|g d\rangle+|d g\rangle),|d d\rangle, \frac{1}{\sqrt{2}}(|p f\rangle+|f p\rangle)\right]$ by the Hamiltonian

$$
H=\left(\begin{array}{cccc}
0 & \frac{\Omega}{\sqrt{2}} & 0 & 0 \\
\frac{\Omega}{\sqrt{2}} & \delta_{L}+E_{|d\rangle} & \frac{\Omega}{\sqrt{2}} & 0 \\
0 & \frac{\Omega}{\sqrt{2}} & 2 \delta_{L}+2 E_{|d\rangle} & U(r) \\
0 & 0 & U(r) & 2 \delta_{L}+E_{|p f\rangle}
\end{array}\right) .
$$

The antisymmetric pair states are not coupled. $E_{|d\rangle}$ is the Stark shift of one atom in the $44 d$ state, $E_{|p f\rangle}$ the shift of the $|p f\rangle$-pair state, and $\delta_{L}$ the detuning of the laser to the $44 d$ state. The Stark shifts are obtained from the calculations in Fig. 2(a). The extension to four resonances, described by a seven-dimensional Hamiltonian, is straightforward.

The experimental spectra can be simulated by numerically solving the Schrödinger equation and summing over all Rydberg populations. In the experiments the Rydbergatom distances cannot be controlled precisely. To account for the Rydberg-atom distribution, the calculated Ramsey spectra for several radii $r$ were weighted and averaged according to a Chandrasekhar distribution [30],

$$
P(r)=e^{-r^{3} / r_{0}^{3}} 3 r^{2} / r_{0}^{3},
$$

describing the nearest-neighbor distribution at the average distance $r_{0}$. These spectra as well as the experimental data were fitted by

$$
\begin{aligned}
N_{\mathrm{Ryd}}\left(\delta_{L}\right)= & N_{0}+a \operatorname{sinc}^{2}\left(\frac{\delta_{L} \tau_{p}}{2}\right) \\
& \times\left[\frac{2 V}{1+V} \cos ^{2}\left(\frac{\delta_{L}\left(\tau_{p}+\tau_{\mathrm{del}}\right)}{2}+\phi\right)+\frac{1-V}{1+V}\right],
\end{aligned}
$$

including seven free-fit parameters, to extract the visibility $V$ and phase $\phi . N_{0}$ describes an offset signal in the detector and $\delta_{L}$ the detuning of the laser relative to the single-atom Rydberg $44 d$ state. 
[1] M. Saffman, T. Walker, and K. Mølmer, Quantum Information with Rydberg Atoms, Rev. Mod. Phys. 82, 2313 (2010).

[2] H. Weimer, M. Müller, I. Lesanowsky, P. Zoller, and H. P. Büchler, A Rydberg Quantum Simulator, Nature Phys. 6, 382 (2010).

[3] B. Butscher, J. Nipper, J. B. Balewski, L. Kukota, V. Bendkowsky, R. Löw, and T. Pfau, Atom-Molecule Coherence for Ultralong-Range Rydberg Dimers, Nature Phys. 6, 970 (2010).

[4] H. Weimer, R. Löw, T. Pfau, and H. P. Büchler, Quantum Critical Behavior in Strongly Interacting Rydberg Gases, Phys. Rev. Lett. 101, 250601 (2008).

[5] S. Westermann, T. Amthor, A.L. de Oliveira, J. Deiglmayr, M. Reetz-Lamour, and M. Weidemüller, Dynamics of Resonant Energy Transfer in a Cold Rydberg Gas, Eur. Phys. J. D 40, 37 (2006).

[6] R. Côté, A. Russell, E. E. Eyler, and P. L. Gould, Quantum Random Walk with Rydberg Atoms in an Optical Lattice, New J. Phys. 8, 156 (2006).

[7] T. Ritz, A. Damjanovic, and K. Schulten, The Quantum Physics of Photosynthesis, Chem. Phys. Chem. 3, 243 (2002).

[8] M. B. Plenio and S.F. Huelga, Dephasing-Assisted Transport: Quantum Networks and Biomolecules, New J. Phys. 10, 113019 (2008).

[9] A. Reinhard, T. Liebisch, B. Knuffman, and G. Raithel, Level Shifts of Rubidium Rydberg States due to Binary Interactions, Phys. Rev. A 75, 032712 (2007).

[10] W. R. Anderson, J. Veale, and T. Gallagher, Resonant Dipole-Dipole Energy Transfer in a Nearly Frozen Rydberg Gas, Phys. Rev. Lett. 80, 249 (1998).

[11] T. Vogt, M. Viteau, J. Zhao, A. Chotia, D. Comparat, and P. Pillet, Dipole Blockade at Förster Resonances in High Resolution Laser Excitation of Rydberg States of Cesium Atoms, Phys. Rev. Lett. 97, 083003 (2006).

[12] P. Bohlouli-Zanjani, J. Petrus, and J. Martin, Enhancement of Rydberg Atom Interactions Using ac Stark Shifts, Phys. Rev. Lett. 98, 203005 (2007).

[13] A. Tauschinsky, C. van Ditzhuijzen, L. Noordam, and H. van den Heuvell, Radio-Frequency-Driven Dipole-Dipole Interactions in Spatially Separated Volumes, Phys. Rev. A 78, 063409 (2008).

[14] I. I. Ryabtsev, D. B. Tretyakov, I. I. Beterov, and V. M. Entin, Observation of the Stark-Tuned Förster Resonance between Two Rydberg Atoms, Phys. Rev. Lett. 104, 073003 (2010).

[15] D. Jaksch, J. I. Cirac, P. Zoller, R. Côté, and M. D. Lukin, Fast Quantum Gates for Neutral Atoms, Phys. Rev. Lett. 85, 2208 (2000).

[16] W. R. Anderson, M. Robinson, J. Martin, and T. Gallagher, Dephasing of Resonant Energy Transfer in a Cold Rydberg Gas, Phys. Rev. A 65, 063404 (2002).
[17] J. Nipper, J. B. Balewski, A. Krupp, B. Butscher, R. Löw, and T. Pfau, Highly Resolved Measurements of StarkTuned Förster Resonances between Rydberg Atoms, Phys. Rev. Lett. 108, 113001 (2012).

[18] M. Reetz-Lamour, T. Amthor, J. Deiglmayr, and M. Weidemüller, Rabi Oscillations and Excitation Trapping in the Coherent Excitation of a Mesoscopic Frozen Rydberg Gas, Phys. Rev. Lett. 100, 253001 (2008).

[19] M. Mei and M. Weitz, Controlled Decoherence in Multiple Beam Ramsey Interference, Phys. Rev. Lett. 86, 559 (2001).

[20] R. Löw, H. Weimer, J. Nipper, J. B. Balewski, B. Butscher, H.P. Büchler, and T. Pfau, An Experimental and Theoretical Guide to Strongly Interacting Rydberg Gases, J. Phys. B 45, 113001 (2012).

[21] R. Heidemann, U. Raitzsch, V. Bendkowsky, B. Butscher, R. Löw, L. Santos, and T. Pfau, Evidence for Coherent Collective Rydberg Excitation in the Strong Blockade Regime, Phys. Rev. Lett. 99, 163601 (2007).

[22] E. A. Donley, N. R. Claussen, S. T. Thompson, and C. E. Wieman, Atom-Molecule Coherence in a Bose-Einstein Condensate, Nature (London) 417, 529 (2002).

[23] I. I. Ryabtsev, D. Tretyakov, I. Beterov, V. Entin, and E. Yakshina, Stark-Tuned Förster Resonance and Dipole Blockade for Two to Five Cold Rydberg Atoms: Monte Carlo Simulations for Various Spatial Configurations, Phys. Rev. A 82, 053409 (2010).

[24] D. Comparat and P. Pillet, Dipole Blockade in a Cold Rydberg Atomic Sample [Invited], J. Opt. Soc. Am. B 27, A208 (2010).

[25] I. Mourachko, D. Comparat, F. de Tomasi, A. Fioretti, P. Nosbaum, V. Akulin, and P. Pillet, Many-Body Effects in a Frozen Rydberg Gas, Phys. Rev. Lett. 80, 253 (1998).

[26] K. R. Younge, A. Reinhard, T. Pohl, P. Berman, and G. Raithel, Mesoscopic Rydberg Ensembles: Beyond the Pairwise-Interaction Approximation, Phys. Rev. A 79, 043420 (2009).

[27] T. A. Johnson, E. Urban, T. Henage, L. Isenhower, D. Yavuz, T. Walker, and M. Saffman, Rabi Oscillations between Ground and Rydberg States with DipoleDipole Atomic Interactions, Phys. Rev. Lett. 100, 113003 (2008).

[28] I. E. Protsenko, G. Reymond, N. Schlosser, and P. Grangier, Operation of a Quantum Phase Gate Using Neutral Atoms in Microscopic Dipole Traps, Phys. Rev. A 65, 052301 (2002).

[29] L. Stryer and R.P. Haugland, Energy Transfer: A Spectroscopic Ruler, Proc. Natl. Acad. Sci. U.S.A. 58, 719 (1967).

[30] S. Chandrasekhar, Stochastic Problems in Physics and Astronomy, Rev. Mod. Phys. 15, 1 (1943). 